\title{
Maintenance plan optimization for a train fleet
}

\author{
K. Doganay \& M. Bohlin \\ Swedish Institute of Computer Science, Kista, Sweden
}

\begin{abstract}
Maintenance planning is an important problem for railways, as well as other application domains that employ machinery with expensive replacements and high downtime costs. In a previous paper, we have developed methods for efficiently finding optimized maintenance schedules for a single unit, and proposed that the maintenance plan should be continuously re-optimized based on the condition of components. However, fleet-level resources, such as the availability of expensive spare parts, have largely been ignored. In this paper, we extend our previous approach by proposing a solution for the fleet level maintenance scheduling problem with spare parts optimization. The new solution is based on a mixed integer linear programming formulation of the problem. We demonstrate the merits of our approach by optimizing instances of maintenance schedules based on maintenance data from railway companies operating in Sweden.

Keywords: maintenance planning, condition based maintenance, optimization, mixed integer programming, railways.
\end{abstract}

\section{Introduction}

Maintenance planning is an important issue, especially for application areas where high cost machinery is used, and when time spent on maintenance disrupts the operation and causes losses, monetary or otherwise. Industry often fears that introducing condition based maintenance (CBM) will lead to more frequent service interventions, which could counter the potential value of implementing CBM. Implementation should therefore be done with care, as the maintenance planning process under CBM needs to be adapted to a much more dynamic situation. We have previously [1] proposed to harvest the full potential value in CBM for rail vehicle maintenance using a combination of condition monitoring and online maintenance planning. A side effect of using this dynamic approach, instead 
of following a traditional cyclic maintenance schedule, is that resources at the maintenance workshop serving a fleet of trains can easily get over-allocated at certain periods. One example is the number of spare parts that need to be available at the service location. Cyclic preventive maintenance usually allows manually building a resource plan that respects the fleet level constraints (e.g. number of spare parts) as the maintenance schedules are relatively regular and cyclic. However, using continuously updated maintenance due dates (from condition monitoring) and frequently re-optimized maintenance schedules for each train makes it impractical to manually construct good fleet level maintenance plans. This can lead to higher resource demands, which is undesirable for expensive spare parts or even unacceptable for some resources. Hence, although each train may have an optimum maintenance plan for itself, the total schedule at fleet level becomes unfeasible.

In this paper, we broaden the perspective by considering a fleet of rail vehicles maintained in a single workshop. Maintenance planning in a long-time perspective is performed to efficiently use the limited spare parts available; for this purpose, we have modeled the planning problem using mixed integer programming (MIP).

\subsection{Vehicle maintenance}

Vehicle maintenance differs from maintenance of stationary equipment in that vehicles are mobile; their current and future location is dependent on the performed and planned jobs for the vehicle. For rail vehicles, planned jobs are usually present in the form of a timetable. Instead of having mobile repair crews visiting the site for maintenance work, the train regularly visits one or several maintenance workshops as a part of the normal duty of the train. In addition, the train dispatching central needs to make sure that the train is indeed sent to the workshop when needed.

Since the freedom to plan maintenance is limited by the assigned timetables, the execution of maintenance actions is also limited to the time intervals when the train is actually in a workshop. These intervals may be (and frequently are) different from the predicted time intervals, since trains are dispatched according to the global train supply and the demand in the network for an operator. In addition, time-consuming setup activities are present in the shunting (movements on a rail yard) of trains to and from the workshop, and parts of the maintenance equipment might be located at other, specialized workshops in the vicinity of the main maintenance workshop. Although not considered in this paper, the layout of the workshop is also important, since there are resource limitations in that a workshop contains a limited number of tracks for vehicles under maintenance. It is also common that tracks have different setups in the form of stationary equipment, such as lifts, graves and power lines. The current state of practice in short-term maintenance planning is manual planning with the aid of computerized maintenance management systems, spreadsheets and possibly project planning tools.

We can predict when each train will be at the maintenance depot from time table data. While this is necessary for planning the current work day (and probably further, typically 1-2 weeks), having very detailed plans for, e.g., three months later is neither necessary nor useful. The reason is simply that the uncertainties in a 
detailed plan are too high in a longer time perspective. For example, one cannot be certain that a particular train will be dispatched to the depot on a specific date and time, as this is dependent on a number of factors, including not only the fleet condition and which vehicles are undergoing maintenance or overhaul, but also other disturbances such as canceled trips due to infrastructure failure or failure to allocate on-board personnel. Moreover, unexpected failures often occur in different components of the train, requiring a visit to the depot earlier than predicted. Although this will invalidate the predicted arrival and departure to the maintenance workshop for the affected trains, it may also lead to an opportunity to perform other maintenance at the same time.

The uncertainties outlined above make it less useful to have detailed plans (in days, hours and minutes) for a planning horizon further than a few weeks. However, this does not mean that planning for more than a few weeks is useless. On the contrary, there is a direct need to plan for the whole maintenance contract period (or at least for a significant part of it), as there may arise situations where too many trains need major maintenance inside the short-term planning horizon, causing a high maintenance load in one month and a corresponding low load in the next.

Another problem arises with spare parts that are maintained offline. An example is a major engine overhaul. As this activity takes about two weeks, it is not performed on the train. Instead, the engine is removed from the train and replaced by a spare engine, so that the train can continue normal operation while the old engine is overhauled in the maintenance workshop. Once the old engine is maintained, it is frequently considered to be as good as new, and can therefore be put into another train later on. Given that it takes two weeks to maintain an engine, the highest number of engine maintenance that occurs in a two week time window during the whole maintenance contract of the whole fleet would equate to the number of spare engines that needs to be available in the maintenance depot. If the fleet maintenance planning do not consider such a long term plan, it would not be able to foresee any conflict caused by too many train units requiring same type of spare parts, or other resources at the maintenance depot.

Our advocacy of CBM and dynamic planning has been met with both high interest and some skepticism by our industrial partners. The main fear lies in the fact that, although each train unit may have a better optimized maintenance schedule, a dynamic and irregular maintenance may lead to infeasible or higher cost schedules for the maintenance depot that serves the whole fleet. In this paper, we consider the spare parts problem as an example of such fleet level constraints and costs. The rest of the paper is organized as follows: section 2 gives an account of related work. In section 3, we explain the optimization model. The method of evaluation is explained and results are reported in section 4. Finally, in section 5, we conclude and discuss future work.

\section{Related work}

The area of optimal maintenance planning and scheduling has been active since the 60s, starting with the seminal work by Barlow and Hunter [2]. Plenty of survey 
papers of the area also exist; fairly recent reviews are given by Budai-Balke $e t$ al. [3] and Nicolai and Dekker [4]. Furthermore, the state of the art in applications of maintenance optimization models is discussed by Dekker and Scarf [5]. More generic mathematical maintenance models are also reviewed by Scarf [6].

In multi-unit maintenance models, the system under consideration consists of several units with identical or individual characteristics regarding failure, costs, setup activities, etc. An overview of multi-unit maintenance models is given by Cho and Parlar [7]. Wildeman et al. [8] discuss maintenance scheduling for a multi-component system with constant co-allocation cost savings, and where deterioration of components is also taken into account.

Rail vehicle maintenance includes the additional complexity of moving equipment, and research in rail vehicle maintenance therefore often includes the associated routing problems. An exception is present in work by Hani et al. [9, 10] who focus on the detailed planning of work performed in the train maintenance facilities only. Cordeau et al. [11] give a survey of models for optimization of train routing and scheduling. In [1, 12], the problem of routing vehicles to the workshop with minimal maintenance costs is solved with the additional sub-problem of grouping maintenance activities such that the number of maintenance occasions is minimized. The problem of determining optimal vehicle routes is NP-hard in general [13], which is why a heuristic method is employed. A related problem has been studied by Anderegg et al. [14], who propose a heuristic routing approach usable in a long-term perspective. Packaging of maintenance is not considered. Maróti and Kroon $[15,16]$ also consider the operational maintenance routing problem without considering maintenance packaging. In [15], a multi-commodity flow model is proposed to solve the problem. In [16], an integer programming formulation is presented, and a shortest path heuristic is proposed to solve the problem for a planning horizon of 1-3 days.

\section{Optimization model}

In this section we define the fleet level planning problem using a discrete time model where each time slot is one week. The problem is formulated as follows.

We are given $n$ identical vehicles $u \in\{1, \ldots, n\}$, each containing $m$ maintenance activities (items) $i \in\{1, \ldots, m\}$ which should be repeated with a period of $T_{i}$ weeks. We use $p$ to denote a spare part type, where $p \in\{1, \ldots, P\}$, and $t$ to denote an occasion, where $t \in\{1, \ldots, H\}$ and $H$ is the schedule length (horizon). The initial condition (used number of weeks) for vehicle $u$ and item $i$ is denoted $O_{u i}$. Each maintenance activity takes $\Delta_{i}$ man-hours to perform and requires the exchange of $\kappa_{i p}$ spare parts of type $p$. Spare parts are repaired offline; repair takes $R_{p}$ weeks for spare part type $p$, and $A_{p}$ spares of type $p$ are available in total. The total cost of having one spare part of type $p$ for one week is $c_{p}$; note that this should include purchase and acquisition costs, transportation costs, and costs due to storage requirements. Maintenance activity $i$ is associated with a fixed $\operatorname{cost} C_{i}$, and at most $k_{t}$ hours of maintenance can be performed in each week $t$.

In addition, the train needs to be shunted before and after each maintenance stop. We model this by including a constant setup $\operatorname{cost} S$ for the activities involved 
in shunting the train. The setup cost is deducted each time at least one maintenance activity, for a single train unit, is performed.

The long-term goal is to plan maintenance for $H$ weeks with minimal cost such that 1) maintenance periods are respected, 2) the maximum maintenance duration in each week is not exhausted, and 3) there are enough spare parts. We only consider preventive maintenance, since corrective maintenance costs can, due to uncertainties on exactly when they "happen", best be estimated outside the model. The number of spare parts needed for corrective maintenance therefore needs to be estimated using statistics, and is not considered further in this paper. Note that we only consider maintenance items for which $T_{i}-O_{i} \leq H$, which implies that the item will definitely be performed within the horizon.

In line with the model proposed by Almgren et al. [17], we use a sequence of binary variables $x_{u i 1}, x_{u i 2}, \ldots, x_{u i H}$ to model maintenance for unit $u$ and item $i$, where $x_{u i t}=1$ indicates that the maintenance item is performed at occasion $t$. The binary variable $y_{u t}$ is used to indicate whether any maintenance is performed for vehicle $u$ at occasion $t$. The variable $U_{p}$ indicates the maximum spare parts needed for type $p$. The fleet-level maintenance planning problem can now be formulated using a mixed integer programming model as follows.

$$
\begin{aligned}
\operatorname{minimize} \underbrace{\sum_{u=1}^{n} \sum_{i=1}^{m} \sum_{t=1}^{H} C_{i} x_{u i t}}_{A} & +\underbrace{\sum_{u=1}^{n} \sum_{t=1}^{H} S y_{u t}}_{B}+\underbrace{H \sum_{p=1}^{P} c_{p} U_{p}}_{C} \\
& +\underbrace{\rho \sum_{u=1}^{n} \sum_{i=1}^{m} \frac{C_{i}}{T_{i}} \sum_{t=H-T_{i}}^{H} t x_{u i t}}_{D}
\end{aligned}
$$

subject to

$$
\begin{array}{rr}
\sum_{j=t}^{t+T_{i}} x_{u i j} \geq 1 & \forall u, i, t \text { where } t \in 1 . . H-T_{i} \\
\sum_{j=1}^{T_{i}-O_{u i}} x_{u i j} \geq 1 & \forall u, i \text { where } T_{i}-O_{u i} \leq H \\
\sum_{u=1}^{n} \sum_{i=1}^{m} \sum_{t^{\prime}=t}^{t+R_{p}} \kappa_{i p} x_{u i t^{\prime}} \leq x_{p i t} & \forall u, i, t \\
U_{p} \leq A_{p} & \forall p, t \in 1 . . H-R_{p} \\
\sum_{u=1}^{n} \sum_{i=1}^{m} \Delta_{u i} x_{u i t} & \leq k_{t} \\
U_{p} \geq 0 \text { real, } x_{u i t}, y_{u t} \text { binary } & \forall p \\
& \forall t \\
& \forall u, i, t, p
\end{array}
$$


The constraints in the problem express that maintenance needs to be performed at least once every $T_{i}$ occasions (2) and at least once within $T_{i}-O_{u i}$ occasions initially (3), that any maintenance at occasion $t$ for unit $u$ indicates that setup costs should be deducted (4), that the number of spare parts needed is greater than the greatest number in service at any single occasion (5) and has a limit (6), and that the amount of labor performed at any occasion should not exceed a given limit (7). The objective (1) of the problem is to minimize costs due to performed maintenance (A), shunting work (B), spare parts (C), and used life at the horizon (D). Maintenance costs of $C_{i}$ is deducted each time activity $i$ is performed, while shunting work costs $S$ on each occasion where at least one activity is performed on a train. For each spare part $p$, a supply of $U_{p}$ units needs to be stored; each spare part costs $c_{p}$ to maintain for one time unit. Finally, a term is needed to discourage solutions in which maintenance is executed well in advance of its deadline. We first assume that neither setup costs nor spare parts costs are deducted after the scheduling horizon; after all, we do not how setup costs and spare parts cost will materialize after this time point. We can then use a weighted penalty (D) of the distance $d_{i}$ of the last activity of type $i$ from the horizon, which for each unit $u$ and item $i$ is $C_{i} / T_{i}$ per time unit of used life. The weight $\rho$, which should be between 0 and 1 , reflect the degree of belief that maintenance will continue after the planning horizon.

To compute $d_{i}$ we need to find the latest activity performed before the horizon. A direct approach would be to count the number of uninterrupted zero-valued binary $x$ variables from the end, but this approach is complicated, and furthermore affects performance negatively. Under the assumptions above, we can do much better using a different approach.

In an optimal schedule, the last $T_{i}$ time units for item $i$ will contain exactly one occurrence of $i$. That at least one occurrence will occur is trivial from (2). Now, assume that there are $x \geq 2$ occurrences of $i$ in the last $T_{i}$ time units in an optimal schedule. The cost contribution of $i$ during the last $T_{i}$ time units is then $C_{i} x+\rho d_{i} C_{i} / T_{i}$, where $d_{i}$ is the used life at the horizon for the last occurrence of $i$, plus some amounts of setup costs (B) and costs due to spare parts usage (C).

Removing the last of the occurrences would decrease direct maintenance costs (A) with $C_{i}$, and at the same time increase penalties (D) with $\rho \delta C_{i} / T_{i}$, where $1 \leq \delta \leq T_{i}-1$ is the distance between the last item and its predecessor. The maximum of the expression can be simplified to $\rho C_{i}(1-1 / T)$ which is less than $C_{i}$ since $\rho \leq 1$. Setup costs and spare parts costs are not increased by removing an activity. Furthermore, constraint (2) would still hold, since there are at least one more occurrence of $i$ before the one being removed, which is within the last $T_{i}$ time units. The other constraints (3-6) would also hold, since the first activity is still present (3), the number of maintenance occurrences is not increased (4), the number of spare parts needed is not increased $(5,6)$ and the amount of work is not increased (7).

Therefore, the schedule can be improved, and the assumption that the schedule is optimal is false. By induction, it is clear that for any optimal schedule, there will 
be at most one activity of type $i$ in the last $T_{i}$ time units. This together with (2) gives us that there is always exactly one activity in the last $T_{i}$ time units for item $i$.

We can now compute the distance $d_{i}$ for the last item of type $i$ to the horizon using the expression

$$
\sum_{t=H-T_{i}}^{H} t x_{u i t},
$$

which uses the fact that exactly one $x_{u i t}$ will be one for $t \in\left\{\left(H-T_{i}\right), \ldots, H\right\}$.

\section{Evaluation}

In order to evaluate the performance of the model explained in section 3 we optimized multiple problem instances using different objective functions, in effect simulating different maintenance strategies, by using ILOG CPLEX 11.2 with AMPL as the modeling language. Computations were carried out on an Intel Xeon 2.83GHz processor, with a 10 minutes CPU time limit for each run.

Maintenance schedules from X11 trains operating in southern Sweden are used as the basis of our scenarios. There are two main scenarios that determine the initial condition of the fleet; the "regular" and the CBM scenario. For both scenarios we decide a random fleet age, and assign an age to each train unit around it, with a standard deviation of six months, again randomly. For the CBM scenario, we further randomize each component's age in the same fashion, by deviating around the particular train's age. The scenario was chosen to simulate the typical dynamic maintenance present when implementing condition based maintenance.

For comparison, we created 50 random samples of both scenarios, and optimized using different objective functions, in effect simulating different maintenance strategies. The block maintenance strategy maintains components as late as possible, and as the maintenance periods of different components are mostly set as multiples of each other, maintenance activities gets planned in blocks (hence the name). This translates to an objective function that includes direct maintenance costs (A) and the used component lifetime (D) in eqn (1). For the second maintenance strategy, the objective function also includes setup costs; (B) in eqn (1). Even though most maintenance activities have a period that is multiple of each other, there exists a few activities that are not, albeit occurring few times in a two year schedule. Such schedules may benefit from optimization, rather than relying on maintenance activities being combined perfectly in a block replacement strategy. We name this second maintenance strategy as optimized without considering spare parts. The third maintenance strategy is optimization considering spare parts, which also includes the total spare part need for the fleet, (C) in eqn (1).

There exists six different major spare parts that are maintained off-line in a separate workshop: two types of bogies, two types of wheelsets, the pantograph and the engine. Each train has two of each types of bogie and wheelsets, four pantographs, and four engines. We used half the price of each part as the cost of having an extra spare part at the maintenance shop for the whole maintenance period. After being removed from a train, maintenance takes three weeks for bogies and two weeks 
for other parts, before it can be safely assumed that the maintained parts can be used in another train. Apart from spare parts, the maintenance schedule consists of 91 different maintenance items with widely varying periodicity. Only one item reoccurs each four weeks, 20 items occur each 8 weeks, another 20 items occur each 24 weeks, and 29 items occur each 72 weeks. Other items have higher periodicity, some as much as 432 weeks, i.e., more than 8 years. In our evaluation, the schedule horizon $H$ that we optimize for is two years. We avoid a bias for new fleets by randomly picking a fleet age for each sample, as described above.

\subsection{Results}

For all instances in the both scenarios (regular and CBM) we optimized using the previously mentioned three strategies. Table 1 lists the average results of 50 samples. For both scenarios, we used the cost of block maintenance strategy as the base and report other values as differences in percentage to that.

Due to the computational limits we imposed (10 minutes CPU time), CPLEX does not find the exact optimum in all cases. For some runs, CPLEX reports an optimality gap as high as $10 \%$, but such gaps occur only when the objective function includes the spare part costs, (C) in eqn (1). In essence, a few of the samples for optimized considering spare parts (last row in table 1) could be further optimized by setting a higher time limit. true only for a few of the cases and included in the results).

Table 1: Optimization results for both scenarios as the average of 50 samples.

\begin{tabular}{lrrrrr}
\hline & \multicolumn{2}{c}{ Regular } & & \multicolumn{2}{c}{ CBM } \\
\cline { 2 - 3 } \cline { 5 - 6 } \cline { 5 - 6 } Block maintenance & 6776241 & $0 \%$ & & 7439488 & $0 \%$ \\
Optimized w/o spares & 6706033 & $-1.04 \%$ & & 7175749 & $-3.55 \%$ \\
Optimized w spares & 5960168 & $-12.04 \%$ & & 5854027 & $-21.31 \%$ \\
\hline
\end{tabular}

In the regular scenario, optimization without considering spare part costs leads to $1.04 \%$ better schedules on average. The difference is higher for the CBM scenario $(3.55 \%)$ because unevenly-aged components in a single train unit gives more opportunities for optimization than the default block maintenance strategy.

Optimization where spare part costs are also considered leads to the biggest gains in both the regular and the CBM scenarios, as expected. The total cost includes spare part costs, so including it in the objective function naturally leads to better optimized schedules. But how much better such schedules would be in practice (i.e., $12.04 \%$ and $21.31 \%$ ) is not so obvious. These values depends heavily on the cost of keeping spare parts available. The cost of having an extra spare part can be extremely low or as high as (or even higher than) the selling price of 
the part, if the particular spare part "gets old" even when not in operation due to oxidation, safety regulations, technology advancement, etc. In our evaluation, we used half of the selling price of each type of major spare part, as the actual operational costs were not available. The optimization results are naturally affected by this estimate, so the quoted percentages for the last optimization strategy ("optimized with spares" in table 1) should not be seen as exact values. Yet, there is still an important implication of the resulting numbers. In both regular and CBM scenarios, when spare parts are also considered in the optimization model, we see that total costs are significantly improved.

\section{Conclusion and future work}

In this paper we described a mixed integer programming model for maintenance schedule optimization. The number of spare parts that needs to be kept in the maintenance depot is also included in the optimization model, as an example of fleet level costs. Our evaluation demonstrate that for each type of scenario, having spare part costs (or any fleet level costs) in the optimization objective reduces the total costs significantly. This way, instead of reducing only the cost of maintaining a single train unit and possibly creating higher-level conflicts, we can optimize for the whole fleet of trains and at the same time satisfy fleet level constraints.

Our model regards preventive maintenance scheduling as a deterministic problem. Although we use maintenance stops due corrective maintenance as an opportunity to schedule preventive maintenance activities, we do not explicitly try to incorporate the possibility of such stops into the schedule from the beginning. In the future, we would like to move into this direction by using stochastic scheduling techniques. It is however not trivial to collect the required data on component failure rates, or make reliable estimates, as such data is often quite sensitive and can be regarded as a trade secret, or even may not exist. We are currently working on collecting and refining relevant data on some key components, such as the pantograph and the break pads, which can be used as input to further stochastic planning efforts.

\section{Acknowledgements}

This work was supported by VINNOVA under grant P32551-1. We would like to thank Ulf Smedbo at EuroMaint Rail AB for providing the maintenance schedules and data used for the evaluation.

\section{References}

[1] Bohlin, M., Forsgren, M., Holst, A., Levin, B., Aronsson, M. \& Steinert, R., Reducing vehicle maintenance using condition monitoring and dynamic planning. Proc. $4^{\text {th }}$ IET Intl. Conf. on Railway Condition Monitoring (RCM'08), 2008.

[2] Barlow, R. \& Hunter, L., Optimum Preventive Maintenance Policies. Oper Res, 8(1), pp. 90-100, 1960. 
[3] Budai-Balke, G., Dekker, R. \& Nicolai, R., A Review of Planning Models for Maintenance and Production. Technical report, Erasmus University Rotterdam, Econometric Institute, 2006. Econometric Institute Report 2006-44.

[4] Nicolai, R. \& Dekker, R., Optimal Maintenance of Multi-Component Systems: a Review. Technical report, Erasmus University Rotterdam, Econometric Institute, 2006. Econometric Institute Report 2006-26.

[5] Dekker, R. \& Scarf, P., On the Impact of Optimisation Models in Maintenance Decision Making: the State of the Art. Rel Eng and Syst Safety, 60(9), pp. 111-119, 1998.

[6] Scarf, P.A., On the Application of Mathematical Models in Maintenance. Eur J Oper Res, 99(3), pp. 493-506, 1997.

[7] Cho, D.I. \& Parlar, M., A survey of maintenance models for multi-unit systems. Eur J Oper Res, 51(1), pp. 1-23, 1991.

[8] Wildeman, R.E., Dekker, R. \& Smit, A.C.J.M., A Dynamic Policy for Grouping Maintenance Activities. Eur J Oper Res, 99(3), pp. 530-551, 1997.

[9] Hani, Y., Amodeo, L., Yalaoui, F. \& Chen, H., Simulation based optimization of a train maintenance facility. J of Intel Manuf, 19(3), pp. 293-300, 2008.

[10] Hani, Y., Chehade, H., Amodeo, L. \& Yalaoui, F., Simulation based optimization of a train maintenance facility model using genetic algorithms. 2006 Intl. Conf. Service Systems and Service Management, volume 1, pp. 513-518, 2006.

[11] Cordeau, J., Toth, P. \& Vigo, D., A Survey of Optimization Models for Train Routing and Scheduling. Transp Sci, 32(4), pp. 380-404, 1998.

[12] Levin, B., Holst, A., Bohlin, M., Steinert, R. \& Aronsson, M., Dynamic maintenance. Proc. $21^{\text {st }}$ Intl. Congress and Exhibition on Condition Monitoring and Diagnostic Engineering Management, 2008.

[13] Erlebach, T., Gantenbein, M., Hürlimann, D., Neyer, G., Pagourtzis, A., Penna, P., Schlude, K., Steinhöfel, K., Taylor, D.S. \& Widmayer, P., On the Complexity of Train Assignment Problems. Proc. 12 th Intl. Symposium on Algorithms and Computation, Springer-Verlag: London, UK, pp. 390-402, 2001.

[14] Anderegg, L., Eidenbenz, S., Gantenbein, M., Stamm, C., Taylor, D.S., Weber, B. \& Widmayer, P., Train Routing Algorithms: Concepts, Design Choices, and Practical. Proceedings of the $5^{\text {th }}$ Workshop on Algorithm Engineering and Experiments, Society for Industrial and Applied Mathematics, pp. 106-118, 2003.

[15] Maróti, G. \& Kroon, L., Maintenance Routing for Train Units: The Transition Model. Transp Sci, 39(4), pp. 518-525, 2005.

[16] Maróti, G. \& Kroon, L., Maintenance Routing for Train Units: The Interchange Model. Comput Oper Res, 34(4), pp. 1121-1140, 2007.

[17] Almgren, T., Andréasson, N., Patriksson, M., Strömberg, A.B. \& Wojciechowski, A., The replacement problem: A polyhedral and complexity analysis. Technical Report Preprint 2009:4, Chalmers University, Göteborg, 2009. 